\title{
Generalized one-loop neutrino mass model with charged particles
}

\author{
Kingman Cheung ${ }^{1,2,3, *}$ and Hiroshi Okada ${ }^{1, \dagger}$ \\ ${ }^{1}$ Physics Division, National Center for Theoretical Sciences, Hsinchu 300, Taiwan \\ ${ }^{2}$ Department of Physics, National Tsing Hua University, Hsinchu 300, Taiwan \\ ${ }^{3}$ Division of Quantum Phases and Devices, School of Physics, Konkuk University, \\ Seoul 143-701, Republic of Korea
}

(Received 3 March 2018; published 20 April 2018)

\begin{abstract}
We propose a radiative neutrino-mass model by introducing 3 generations of fermion pairs $E^{-(N+1) / 2} E^{+(N+1) / 2}$ and a couple of multicharged bosonic doublet fields $\Phi_{N / 2}, \Phi_{N / 2+1}$, where $N=1$, $3,5,7,9$. We show that the models can satisfy the neutrino masses and oscillation data, and are consistent with lepton-flavor violations, the muon anomalous magnetic moment, the oblique parameters, and the beta function of the $U(1)_{Y}$ hypercharge gauge coupling. We also discuss the collider signals for various $N$, namely, multicharged leptons in the final state from the Drell-Yan production of $E^{-(N+1) / 2} E^{+(N+1) / 2}$. In general, the larger the $N$ the more charged leptons will appear in the final state.
\end{abstract}

DOI: 10.1103/PhysRevD.97.075027

\section{INTRODUCTION}

Nonzero neutrino mass is the most intriguing evidence for physics beyond the standard model (SM). The scale of neutrino mass $(\sim 0.1 \mathrm{eV})$ is at least 12 orders of magnitude smaller than the electroweak scale. In order to explain such a tiny neutrino mass, various mechanisms have been proposed to explain the phenomena. Conventionally, the seesaw mechanism [1] with a high seesaw scale $\left(\sim 10^{11-12} \mathrm{GeV}\right)$ is one of the most natural mechanisms to generate such a tiny mass. However, such a high seesaw scale offers no immediate laboratory tests. Therefore, a number variety of seesaw models appeared afterwards, e.g., inverse seesaw [2].

Another category of models is the radiatively generated neutrino-mass models, in which the smallness of neutrino mass is achieved by loop suppression. A few of the earliest models are the Zee model [3], Babu model [4], and Ma model [5]. In general, it requires new particles running in the $\operatorname{loop}(\mathrm{s})$ of the neutrino-mass generating diagrams. These new particles can be light enough to be produced at colliders, thus offering immediate tests for the model. They could also be relevant to explain other phenomena,

\footnotetext{
cheung@phys.nthu.edu.tw

†macokada3hiroshi@cts.nthu.edu.tw
}

Published by the American Physical Society under the terms of the Creative Commons Attribution 4.0 International license. Further distribution of this work must maintain attribution to the author(s) and the published article's title, journal citation, and DOI. Funded by SCOAP ${ }^{3}$. such as dark matter, lepton-flavor violations, muon anomalous magnetic moment, etc.

In a previous work, we proposed a simple extension of the SM by introducing 3 generations of doubly charged fermion pairs $E^{--} E^{++}$and three multicharged bosonic fields $k^{++}, \Phi_{3 / 2}, \Phi_{5 / 2}$, in which $\Phi$ are the $S U(2)$ doublet fields and $k^{++}$is a singlet field [6]. The model can explain the small neutrino masses and oscillations, muon anomalous magnetic moment, and is consistent with the lepton-flavor violations and the oblique parameters.

Here in this work we generalize the model to $E^{-\frac{N+1}{2}} E^{+\frac{N+1}{2}}$ and $\Phi_{N / 2}, \Phi_{N / 2+1}$, with $N=1,3,5,7,9$. The previous work [6] corresponds to the case of $N=3$. The generalization is indeed nontrivial. Especially, for the case of $N=1$ in which a $Z_{2}$ parity is required to distinguish between the Higgs doublet $H$ and the doublet $\Phi_{1 / 2}$. The $Z_{2}$ assignment thus gives rise to a lightest $Z_{2^{-}}$ odd particle, which is stable and can be a dark matter candidate. We will explore the dark matter phenomenology of $N=1$ case. The other cases share some similar features as $N=3$, but they do have different features that deserve separate discussion. In general, a larger $N$ would rise to a final state with more charged leptons for DrellYan production of $E^{+(N+1) / 2} E^{-(N+1) / 2}$.

This paper is organized as follows. In Sec. II, we review the model and describe the constraints. In Sec. III, we describe the physics for each $N$. In Sec. IV, we present the numerical analyses and valid parameter space for each $N$. We discuss the collider signals in Sec. V and conclude in Sec. VI. 
TABLE I. Charge assignments of new fields under $S U(2)_{L} \times$ $U(1)_{Y}$ with $1 \leq N$ and $N^{\prime} \equiv N+2$ with odd number, where all the new fields are color singlet.

\begin{tabular}{lcccccc}
\hline \hline & $L_{L}$ & $e_{R}$ & $E_{L / R}$ & $H$ & $\Phi_{N / 2}$ & $\Phi_{N^{\prime} / 2}^{\prime}$ \\
\hline$S U(2)_{L}$ & $\mathbf{2}$ & $\mathbf{1}$ & $\mathbf{1}$ & $\mathbf{2}$ & $\mathbf{2}$ & $\mathbf{2}$ \\
$U(1)_{Y}$ & $-\frac{1}{2}$ & -1 & $-\frac{N+1}{2}$ & $\frac{1}{2}$ & $\frac{N}{2}$ & $\frac{N^{\prime}}{2}$ \\
\hline \hline
\end{tabular}

\section{MODEL SETUP AND CONSTRAINTS WITH COMMON PART}

In our set up of the model, we introduce three families of doubly charged fermions $E^{1}$ and two types of new bosons $\Phi_{N / 2}$ and $\Phi_{N^{\prime} / 2}$ with $N^{\prime} \equiv N+2$, as shown in Table I. Notice here that one has to impose an additional symmetry such as $Z_{2}$ to discriminate between $\Phi_{N / 2}$ and $H$ only in case of $N=1$, as we will see later. The renormalizable Lagrangian in the lepton sector and the Higgs potential are given by

$$
\begin{aligned}
-\mathcal{L}_{Y}= & \left(y_{\ell}\right)_{i i} \bar{L}_{i} H P_{R} e_{i}+f_{i a} \bar{L}_{i} \Phi_{N / 2} P_{R} E_{a} \\
& +g_{i a} \bar{L}_{i} \cdot \Phi_{N^{\prime} / 2}^{\prime *} P_{R} E_{a}^{c}+M_{E_{a}} \bar{E}_{a} E_{a}+\text { H.c., } \\
V= & \mu_{H}^{2}|H|^{2}+\mu_{\Phi}^{2}\left|\Phi_{N / 2}\right|^{2}+\mu_{\Phi^{\prime}}^{2}\left|\Phi_{N^{\prime} / 2}^{\prime 2}\right| \\
& +\left[\lambda_{0}\left(H^{T} \cdot \Phi_{N / 2}\right)\left(H^{T} \cdot \Phi_{N^{\prime} / 2}^{\prime *}\right)+\text { c.c. }\right] \\
& +\left[\lambda_{0}^{\prime}\left(\Phi_{N^{\prime} / 2}^{\prime \dagger} \Phi_{N / 2}\right)_{3}\left(H^{T} H\right)_{3}+\text { c.c. }\right] \\
& +\lambda_{H}|H|^{4}+\lambda_{\Phi}\left|\Phi_{N / 2}\right|^{4}+\lambda_{\Phi^{\prime}}\left|\Phi_{N^{\prime} / 2}^{\prime}\right|^{4} \\
& +\lambda_{\Phi \Phi^{\prime}}\left|\Phi_{N / 2}\right|^{2}\left|\Phi_{N^{\prime} / 2}^{\prime}\right|^{2}+\text { H.c., }
\end{aligned}
$$

where $(i, a)=1-3$ are generation indices and the multiplication symbol represents $i \sigma_{2}$ with $\sigma_{2}$ being the second Pauli matrix. The first term in the Yukawa Lagrangian, which is assumed to be diagonal for convenience, provides the masses for the charged leptons $\left(m_{\ell_{i}} \equiv y_{\ell_{i i}} v / \sqrt{2}\right)$ by developing a nonzero vacuum expectation value (VEV) of $H$, which is symbolized by $\langle H\rangle \equiv v / \sqrt{2}$. Also, we work in the basis where all the coefficients are real and positive for simplicity hereafter. We can parametrize the scalar fields as

$$
\Phi_{N / 2}=\left[\begin{array}{l}
\phi^{\frac{N+1}{2}} \\
\phi^{\frac{N-1}{2}}
\end{array}\right], \quad \Phi_{N^{\prime} / 2}^{\prime}=\left[\begin{array}{c}
\phi^{\frac{N+3}{2}} \\
\phi^{\frac{N+1}{2}}
\end{array}\right],
$$

where the superscript for each component represents the electric charges. Due to the $\lambda_{0}^{\left({ }^{\prime}\right)}$ term in Eq. (2.1), the two $\frac{N+1}{2}$-charged bosons in basis of $\left(\phi^{\frac{N+1}{2}}, \phi^{\frac{N+1}{2}}\right)$ mix with each other. Their mixing matrix and mass eigenstates are defined as

\footnotetext{
${ }^{1}$ In order to minimally reproduce the neutrino oscillation data, two families of $E$ are enough. In this case, a massless neutrino is induced.
}

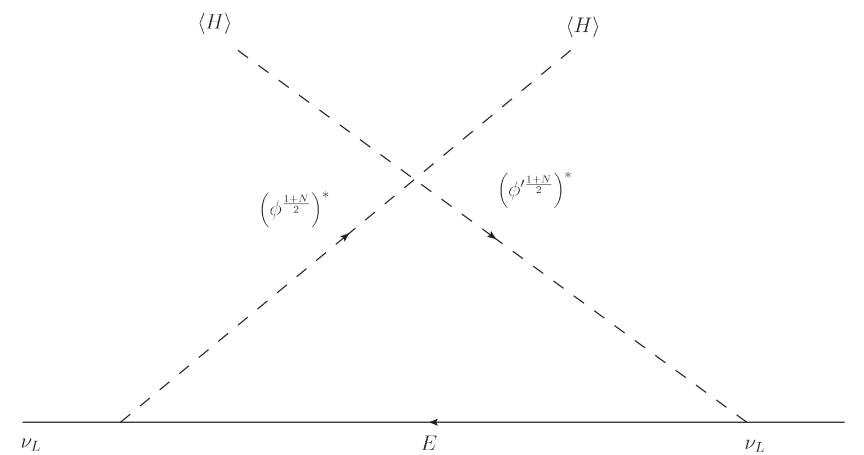

FIG. 1. One-loop diagrams for generating the neutrino mass matrix.

$$
\left[\begin{array}{c}
\phi^{\frac{N+1}{2}} \\
\phi^{\prime \frac{N+1}{2}}
\end{array}\right]=\sum_{a=1-2} O_{i a} H_{a}, \quad O \equiv\left[\begin{array}{cc}
c_{\theta} & -s_{\theta} \\
s_{\theta} & c_{\theta}
\end{array}\right] .
$$

Therefore one can redefine these bosons as the mass eigenstates as follows:

$\phi^{\frac{N+1}{2}}=c_{\theta} H_{1}-s_{\theta} H_{2}, \quad \phi^{\frac{N+1}{2}}=s_{\theta} H_{1}+c_{\theta} H_{2}$,

where we have used the short-hand notation: $H_{i} \equiv H_{i}^{\frac{N+1}{2}}$ $(i=1,2)$, and their masses to be $m_{H_{i}^{\frac{N+1}{2}}} \equiv m_{H_{i}}$.

\section{A. Neutrino mixing}

The active neutrino mass matrix $M_{\nu}$ is given at one-loop level via doubly charged particles in Fig. 1, and its formula is given by [6]

$$
\begin{gathered}
-\left(M_{\nu}\right)_{i j}=\frac{2 s_{2 \theta}}{(4 \pi)^{2}} \sum_{a=1}^{3} f_{i a} M_{a} g_{a j}^{T} F_{I}\left(M_{a}, H_{1}, H_{2}\right)+(f \leftrightarrow g) \\
\quad \equiv f_{i a} R_{a} g_{a j}^{T}+g_{i a} R_{a} f_{a j}^{T} \\
F_{I}\left(m_{a}, m_{b}, m_{c}\right) \\
=\frac{m_{a}^{2} m_{b}^{2} \ln \left(\frac{m_{a}}{m_{b}}\right)+m_{a}^{2} m_{c}^{2} \ln \left(\frac{m_{a}}{m_{c}}\right)+m_{b}^{2} m_{c}^{2} \ln \left(\frac{m_{b}}{m_{c}}\right)}{\left(m_{a}^{2}-m_{b}^{2}\right)\left(m_{a}^{2}-m_{c}^{2}\right)}
\end{gathered}
$$

The mass matrix $M_{\nu}$ is diagonalized by the neutrino mixing matrix $V_{\mathrm{MNS}}$ as $M_{\nu}=V_{\mathrm{MNS}} D_{\nu} V_{\mathrm{MNS}}^{T}$ with $D_{\nu} \equiv\left(m_{\nu_{1}}, m_{\nu_{2}}, m_{\nu_{3}}\right)$. Then one can parametrize Yukawa coupling in terms of arbitrary antisymmetric matrix with complex values $A ;\left(A+A^{T}=0\right)$, as follows [7]:

$$
\begin{aligned}
& f=-\frac{1}{2}\left[V_{\mathrm{MNS}} D_{\nu} V_{\mathrm{MNS}}^{T}+A\right]\left(g^{T}\right)^{-1} R^{-1}, \\
& g=-\frac{1}{2}\left[V_{\mathrm{MNS}} D_{\nu} V_{\mathrm{MNS}}^{T}+A\right]^{T}\left(f^{T}\right)^{-1} R^{-1} .
\end{aligned}
$$

In the numerical analysis, we shall use the latter relation for convenience and use the data in the global analysis [8]. 
It is worthwhile to describe the differences between the Ma model and our model. Once we assign the lepton number +2 for $\Phi_{N^{\prime} / 2}^{\prime}$ and zero for other bosons, the lepton number is explicitly broken at the $\lambda_{0}$ and $\lambda_{0}^{\prime}$ terms by 2 units. On the other hand, if all the bosons were assigned zero charges under the lepton number, then the breaking of the lepton number would occur at the Yukawa coupling term $g$. Here, we suppose that the extra vector fermions $E$ has -1 charge under the lepton number, which is the same as the SM charged leptons. The major difference between our model and the Ma model is that the lepton number in the Ma model is violated via the mass term of the righthanded Majorana neutrinos while in our model it is violated via the boson mass or the Yukawa term.

\section{B. Lepton flavor violations and muon anomalous magnetic moment}

The Yukawa terms of $(f, g)$ give rise to $\ell_{i} \rightarrow \ell_{j} \gamma$ processes at one-loop level. The branching ratio is given by

$$
B\left(\ell_{i} \rightarrow \ell_{j} \gamma\right) \approx \frac{48 \pi^{3} \alpha_{\mathrm{em}}}{G_{\mathrm{F}}^{2}} C_{i j}\left|\mathcal{M}_{i j}\right|^{2}
$$

where $G_{\mathrm{F}} \approx 1.166 \times 10^{-5} \mathrm{GeV}^{-2}$ is the Fermi constant, $\alpha_{\mathrm{em}}\left(m_{Z}\right) \approx 1 / 128.9$ is the fine-structure constant [9], $C_{21} \approx 1, C_{31} \approx 0.1784$, and $C_{32} \approx 0.1736 . \mathcal{M}\left(=\mathcal{M}_{f}+\mathcal{M}_{g}\right)$ is formulated as

$$
\begin{aligned}
\left(\mathcal{M}_{f}\right)_{i j} \approx & -\sum_{a=1-3} \frac{f_{j a} f_{a i}^{\dagger}}{(4 \pi)^{2}}\left[\frac{N-1}{2} F_{l f v}\left(M_{E_{a}}, m_{\phi}\right)\right. \\
& \left.+\frac{N+1}{2} F_{l f v}\left(m_{\phi}, M_{E_{a}}\right)\right], \\
\left(\mathcal{M}_{g}\right)_{i j} \approx & \sum_{a=1-3} \frac{g_{j a} g_{a i}^{\dagger}}{(4 \pi)^{2}}\left[\frac{N+3}{2} F_{l f v}\left(M_{E_{a}}, m_{\phi^{\prime}}\right)\right. \\
& \left.+\frac{N+1}{2} F_{l f v}\left(m_{\phi^{\prime}}, M_{E_{a}}\right)\right], \\
F_{l v s}\left(m_{1}, m_{2}\right) & 2 m_{1}^{6}+3 m_{1}^{4} m_{2}^{2}-6 m_{1}^{2} m_{2}^{4}+m_{2}^{6}+12 m_{1}^{4} m_{2}^{2} \ln \left[\frac{m_{2}}{m_{1}}\right] \\
\approx & \frac{12\left(m_{1}^{2}-m_{2}^{2}\right)^{4}}{}
\end{aligned}
$$

where we have simplified the notation $\phi \equiv \phi^{\frac{N-1}{2}}$, and $\phi^{\prime} \equiv \phi^{\prime \frac{N+3}{2}}$, and assumed $m_{\ell_{\tau}} \ll M_{E_{a}}, m_{\phi}, m_{\phi^{\prime}}$ in the equations above. ${ }^{2}$ The current experimental upper bounds are given by $[10,11]$

\footnotetext{
${ }^{2}$ It implies that the mass difference between $m_{1}$ and $m_{2}$ should be greater than the order of $m_{\ell_{\tau}}$.
}
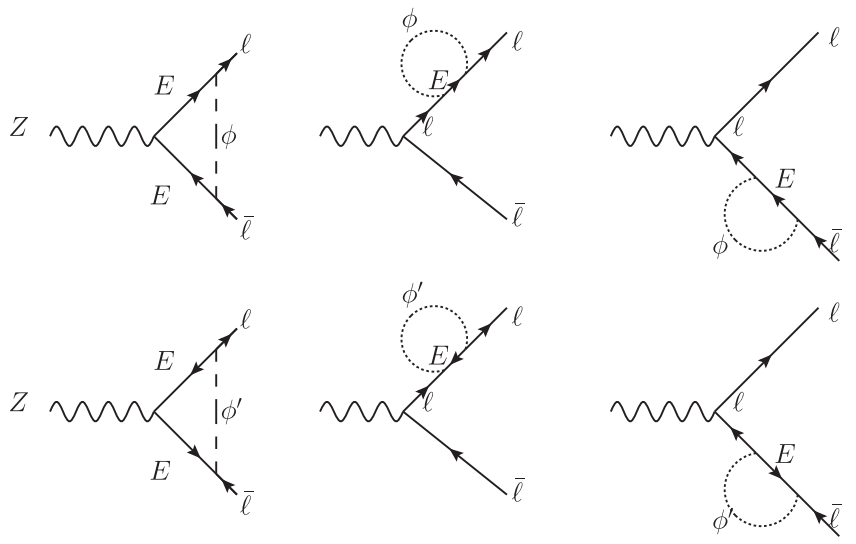

FIG. 2. Feynman diagrams for $Z \rightarrow \ell_{i} \bar{\ell}_{j}$, where upper diagrams represent the contributions for $f$, while the down ones for $g$.

$$
\begin{aligned}
& B(\mu \rightarrow e \gamma) \leq 4.2 \times 10^{-13}, \quad B(\tau \rightarrow \mu \gamma) \leq 4.4 \times 10^{-8}, \\
& B(\tau \rightarrow e \gamma) \leq 3.3 \times 10^{-8} .
\end{aligned}
$$

The muon anomalous magnetic moment $\left(\Delta a_{\mu}\right)$ : We can also estimate the muon anomalous magnetic moment through $\mathcal{M}$, which is given by

$$
\Delta a_{\mu} \approx-m_{\mu}^{2} \mathcal{M}_{22}
$$

The $3.3 \sigma$ deviation from the SM prediction is $\Delta a_{\mu}=$ $(26.1 \pm 8) \times 10^{-10}$ [12] with a positive value. Obviously, $f$ contributes to the $\Delta a_{\mu}$ positively, while $g$ does negatively. To achieve the agreement with the experimental result, one has to enhance the contributions from $f$ compared to those from $g$.

\section{Flavor-changing leptonic $Z$ boson decays}

In this section, we consider the $Z$ boson decay into two charged leptons with different flavors through the Yukawa terms $f$ and $g$ at the one-loop level [13]. Since some components of $f$ and $g$ are expected to be large so as to obtain a sizable $\Delta a_{\mu}$, the experimental bounds on leptonflavor violating $Z$ boson decays could be of concern at the one loop level. First of all, the relevant Lagrangian is given by

$$
\begin{aligned}
\mathcal{L}_{\text {kin }} \sim & \frac{g_{2}}{c_{w}}\left[\bar{\ell} \gamma^{\mu}\left(-\frac{1}{2}+s_{w}^{2}\right) \ell+\frac{N+1}{2} s_{W}^{2} \bar{E} \gamma^{\mu} E\right] Z_{\mu} \\
& +\left[f_{i a} \bar{\ell}_{i} P_{R} E_{a} \phi-g_{i a} \bar{\ell}_{i} P_{R} E_{a}^{c} \phi^{\prime *}+\text { H.c. }\right],
\end{aligned}
$$

where $s(c)_{W} \equiv \sin (\cos ) \theta_{W} \sim 0.23$ stands for the sine (cosine) of the Weinberg angle. Combining all the diagrams in Fig. 2, the ultraviolet divergence cancels out and only the finite part remains [13]. The resulting form is given by 
$\mathrm{BR}\left(Z \rightarrow \ell_{i}^{-} \ell_{j}^{+}\right)=\frac{G_{F}}{3 \sqrt{2} \pi} \frac{m_{Z}^{3} s_{w}^{4}(N+1)^{2}}{4\left(16 \pi^{2}\right)^{2} \Gamma_{Z}^{\mathrm{tot}}}\left|\sum_{a=1}^{3} f_{i a} f_{j a}^{*}\left[F_{2}\left(E_{a}, \phi\right)+F_{3}\left(E_{a}, \phi\right)\right]+\sum_{b=1}^{3} g_{i b} g_{j b}^{*}\left[F_{2}\left(E_{b}, \phi^{\prime}\right)+F_{3}\left(E_{b}, \phi^{\prime}\right)\right]\right|^{2}$,

where $i \neq j$,

$$
\begin{aligned}
& F_{2}(a, b)=\int_{0}^{1} d x(1-x) \ln \left[(1-x) m_{a}^{2}+x m_{b}^{2}\right] \\
& F_{3}(a, b)=\int_{0}^{1} d x \int_{0}^{1-x} d y \frac{(x y-1) m_{Z}^{2}+\left(m_{a}^{2}-m_{b}^{2}\right)(1-x-y)-\Delta \ln \Delta}{\Delta},
\end{aligned}
$$

with $\Delta \equiv-x y m_{Z}^{2}+(x+y)\left(m_{a}^{2}-m_{b}^{2}\right)+m_{b}^{2}$ and the total $Z$ decay width $\Gamma_{Z}^{\text {tot }}=2.4952 \pm 0.0023 \mathrm{GeV}$ [14]. From Eqs. (2.9), (2.10) and (2.15), one finds that the same combinations of Yukawa couplings $f_{i a} f_{j a}^{*}$ and $g_{i a} g_{j a}^{*}$ appear in $\ell_{i} \rightarrow \ell_{j} \gamma$ and $Z \rightarrow \ell_{i} \ell_{j}$. Note that the predictions for $\operatorname{BR}(Z \rightarrow \mu \tau)$ can be rather large and tested in future experiments such as Giga-Z [15], due to different properties of loop functions [13]. The current bounds on the leptonflavor-changing $Z$ boson decay branching ratios at 95\% C.L. are given by [14]

$$
\begin{aligned}
& \operatorname{BR}\left(Z \rightarrow e^{ \pm} \mu^{\mp}\right)<1.7 \times 10^{-6}, \\
& \operatorname{BR}\left(Z \rightarrow e^{ \pm} \tau^{\mp}\right)<9.8 \times 10^{-6}, \\
& \operatorname{BR}\left(Z \rightarrow \mu^{ \pm} \tau^{\mp}\right)<1.2 \times 10^{-5} .
\end{aligned}
$$

We include these constraints in the global analysis.

\section{Oblique parameters}

In order to estimate the testability via collider physics, we have to consider the oblique parameters that restrict the mass hierarchy between each of component $\Phi_{N / 2}$ and $\Phi_{N^{\prime} / 2}$.

Here we focus on the new physics contributions to the $S$ and $T$ parameters in the case of $\Delta U=0$. Then $\Delta S$ and $\Delta T$ are defined as

$$
\begin{aligned}
& \Delta S=\left.16 \pi \frac{d}{d q^{2}}\left[\Pi_{33}\left(q^{2}\right)-\Pi_{3 Q}\left(q^{2}\right)\right]\right|_{q^{2} \rightarrow 0}, \\
& \Delta T=\frac{16 \pi}{s_{W}^{2} m_{Z}^{2}}\left[\Pi_{ \pm}(0)-\Pi_{33}(0)\right],
\end{aligned}
$$

where $s_{W}^{2} \approx 0.23$ is the Weinberg angle and $m_{Z}$ is the $Z$ boson mass. The loop factors $\Pi_{33,3 Q, \pm}\left(q^{2}\right)$ are calculated from the one-loop vacuum-polarization diagrams for $Z$ and $W^{ \pm}$bosons, which are respectively given by $[16,17]$

$$
\begin{aligned}
\Pi_{33}\left(q^{2}\right)= & \frac{1}{2(4 \pi)^{2}}\left[G(\phi, \phi)+G\left(H_{\alpha}, H_{\alpha}\right)+G\left(\phi^{\prime}, \phi^{\prime}\right)\right. \\
& \left.-H(\phi)-H\left(H_{\alpha}\right)-H\left(\phi^{\prime}\right)\right],
\end{aligned}
$$

$$
\begin{aligned}
\Pi_{3 Q}\left(q^{2}\right)= & \frac{1}{(4 \pi)^{2}}\left[-m G\left(q^{2}, \phi, \phi\right)+(m+2) G\left(q^{2}, \phi^{\prime}, \phi^{\prime}\right)\right. \\
& +m H(\phi)-(m+2) H\left(\phi^{\prime}\right) \\
& +(m+1)\left[( c _ { \theta } ^ { 2 } - s _ { \theta } ^ { 2 } ) \left(G\left(q^{2}, H_{1}, H_{1}\right)\right.\right. \\
& \left.-G\left(q^{2}, H_{2}, H_{2}\right)\right)-2 c_{\theta} s_{\theta}\left(G\left(q^{2}, H_{1}, H_{2}\right)\right. \\
& \left.\left.+G\left(q^{2}, H_{2}, H_{1}\right)\right)\right] \\
& \left.-(m+1)\left(c_{\theta}^{2}-s_{\theta}^{2}\right)\left(H\left(H_{1}\right)-H\left(H_{2}\right)\right)\right],
\end{aligned}
$$

$$
\begin{aligned}
\Pi_{ \pm}\left(q^{2}\right)= & \frac{1}{(4 \pi)^{2}}\left[c_{\theta}^{2} G\left(q^{2}, \phi, H_{1}\right)+s_{\theta}^{2} G\left(q^{2}, \phi, H_{2}\right)\right. \\
& +s_{\theta}^{2} G\left(q^{2}, H_{1}, \phi^{\prime}\right)+c_{\theta}^{2} G\left(H_{2}, \phi^{\prime}\right) \\
& \left.-\frac{1}{2}\left[H(\phi)+H\left(\phi^{\prime}\right)+H\left(H_{1}\right)+H\left(H_{2}\right)\right]\right],
\end{aligned}
$$

$$
\begin{aligned}
G\left(q^{2}, m 1, m 2\right) \equiv & \int[d X]_{2}\left[-q^{2} x(1-x)+x m_{1}^{2}+y m_{2}^{2}\right] \\
& \times\left(\Upsilon+1-\ln \left[-\frac{q^{2}}{m_{1}^{2}} x(1-x)+x+y \frac{m_{2}^{2}}{m_{1}^{2}}\right]\right),
\end{aligned}
$$

where $H(m) \equiv m^{2}[\Upsilon+1],{ }^{3} \int[d X]_{2} \equiv \int_{0}^{1} d x d y \delta(1-x-y)$, $\Upsilon \equiv \frac{1}{\epsilon}-\gamma-\ln (4 \pi), m \equiv \frac{N-1}{2}$ is the electric charge, and $\alpha(=1,2)$ should be summed up. Fixing $\Delta U=0$, the experimental bounds on $\Delta S$ and $\Delta T$ are given by [18]

$\Delta S=(0.05 \pm 0.09), \quad \Delta T=(0.08 \pm 0.07)$,

with a correlation coefficient of +0.91 . The $\Delta \chi^{2}$ can be calculated as [19]

$$
\begin{aligned}
\Delta \chi^{2}= & \sum_{(i, j)=1,2}(\Delta S-0.05, \Delta T-0.08) \\
& \times\left[\begin{array}{cc}
718.19 & -840.28 \\
-840.28 & 1187.2
\end{array}\right]\left[\begin{array}{c}
\Delta S-0.05 \\
\Delta T-0.08
\end{array}\right],
\end{aligned}
$$

\footnotetext{
${ }^{3}$ Notice here that $H(m)$ does not depend on the referenced energy $q^{2}$ that arises from contact interacting loop functions.
} 
and we impose the $99 \%$ confidence level limit that corresponds to $\Delta \chi^{2}=9.210$ in our numerical analysis.

\section{E. Beta function of $g_{Y}$}

Here we estimate the effective energy scale by evaluating the Landau pole for $g_{Y}$ in the presence of new exotic fields with nonzero multiple hypercharges. Each contribution of the new beta function of $g_{Y}$ from one $S U(2)_{L}$ doublet fermion or boson with $\pm N / 2$ hypercharge is given by [20]

$$
\Delta_{s} b_{Y}^{f}=\frac{3}{5} \times \frac{4}{3} \times\left(\frac{N}{2}\right)^{2}, \quad \Delta_{s} b_{Y}^{b}=\frac{3}{5} \times \frac{2}{3} \times\left(\frac{N}{2}\right)^{2},
$$

where the superscript of $\Delta b$ represents the fermion (f) or the boson (b), respectively. Similarly, the contribution to the beta function from one $S U(2)_{L}$ singlet boson with $\pm N / 2$ hypercharge (= electric charge) is given by

$$
\Delta_{d} b_{Y}^{f}=\frac{3}{5} \times \frac{2}{3} \times\left(\frac{N}{2}\right)^{2}, \quad \Delta_{d} b_{Y}^{b}=\frac{3}{5} \times \frac{1}{3} \times\left(\frac{N}{2}\right)^{2},
$$

where $3 / 5$ is the rescaled coefficient. Here let us include a doubly charged gauge-singlet boson $k^{ \pm \pm}$in order to make appropriate decays into the SM fields as we will discuss later. ${ }^{4}$ Then one finds the energy evolution of the gauge coupling $g_{Y}$ as [21]

$$
\begin{aligned}
\frac{1}{g_{Y}^{2}(\mu)}= & \frac{1}{g_{Y}^{2}\left(m_{\mathrm{in}}\right)}-\frac{b_{Y}^{\mathrm{SM}}}{(4 \pi)^{2}} \ln \left[\frac{\mu^{2}}{m_{\mathrm{in}}^{2}}\right] \\
& -\theta\left(\mu-m_{\text {thres }_{f}}\right) \frac{\Delta b_{Y}^{f}}{(4 \pi)^{2}} \ln \left[\frac{\mu^{2}}{m_{\text {thres }_{f}}^{2}}\right] \\
& -\theta\left(\mu-m_{\text {thres }_{b}}\right) \frac{\Delta b_{Y}^{b}}{(4 \pi)^{2}} \ln \left[\frac{\mu^{2}}{m_{\text {thres }_{b}}^{2}}\right],
\end{aligned}
$$

where $\mu$ is a reference energy scale, $b_{Y}^{\mathrm{SM}}=41 / 6, \Delta b_{Y}^{f}=$ $3 \Delta_{s} b_{Y}^{f}=(N+1)^{2} / 2, \quad \Delta b_{Y}^{b}=\Delta_{d} b_{Y}^{b}\left(\Phi_{N / 2}\right)+\Delta_{d} b_{Y}^{b}\left(\Phi_{N^{\prime} / 2}^{\prime}\right)+$ $\Delta_{s} b_{Y}^{b}\left(k^{++}\right)=\left(N^{2}+2 N+6\right) / 3$, and we assume that $m_{\text {in }}\left(=m_{Z}\right)<m_{\text {thres }_{f}}=m_{\text {thres }_{b}}=500 \mathrm{GeV}$, where the threshold masses for exotic fermions and bosons are $m_{\text {thres }_{f}}$ and $m_{\text {thres }_{b}}$, respectively. The resulting running of $g_{Y}(\mu)$ versus the scale $\mu$ is shown in Fig. 3 for each of $N=1,3,5,7,9$, 11 , where we analyze $N$ up to 9 in our global analyses. ${ }^{5}$ In the cases of $N=1,3$, perturbativity allows the cutoff scale up to Planck mass. However, the case of $N=5,7,9,11$ is

\footnotetext{
${ }^{4} k^{++}$is valid for $N=3,5,7,9$ to make appropriate decays, where it is not needed for $N=1$ due to the existence of the DM candidate but an additional symmetry such as $Z_{2}$ is needed.

${ }^{5}$ When one considers the case of $11 \leq N$, one has to introduce more particles in order to make appropriate decays of exotic particles into the SM particles. For example, the minimum extension in the case of $N=11$ is to add a gauge-singlet quartic-charged boson $h^{ \pm \pm \pm \pm}$. In this case, the valid scale (= at the Landau pole) decreases down to $10^{4} \mathrm{GeV}$.
}

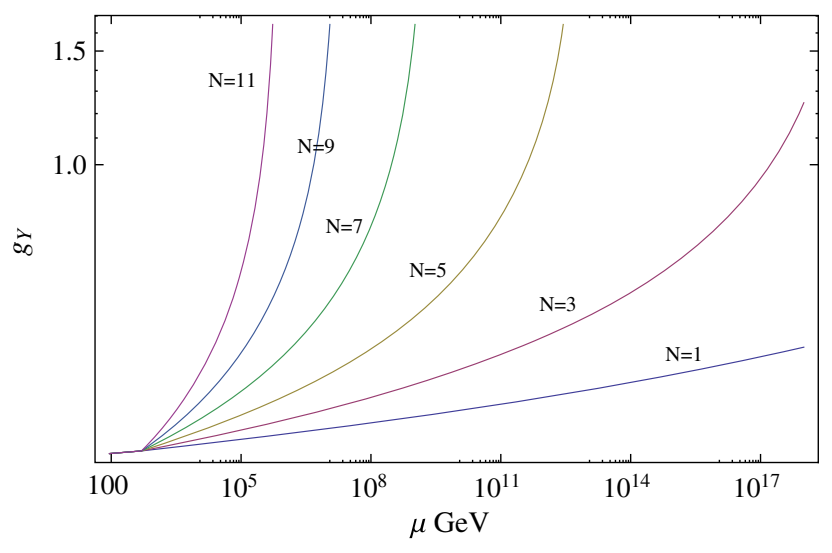

FIG. 3. The running of $g_{Y}$ in terms of a reference energy of $\mu$, depending on each of $N=1,3,5,7,9,11$.

valid up to around $3 \times 10^{11} \mathrm{GeV}, 3 \times 10^{8} \mathrm{GeV}, 3 \times 10^{5} \mathrm{GeV}$, and $10^{5} \mathrm{GeV}$, respectively. Notice here that $g_{2}$ running is almost the same the one of SM.

\section{PHYSICS OF EACH $N$}

Here we investigate each of $N(1,3,5,7,9)$ below, where we introduce a gauge-singlet doubly charged boson $k^{++}$for $3 \leq N$. We assume that the mixing between $k^{++}$and a component of a doubly charged boson with an isospin doublet $\Phi_{N / 2}$ and/or $\Phi_{N^{\prime} / 2}^{\prime}$ are small enough to be neglected for the neutrino oscillations, LFVs, and $\Delta a_{\mu}$, although the mixing could play an important role in appropriately making exotic fields decay into the SM fermions.

\section{A. $N=1$}

The case of $N=1$ is different from other $N$ 's, because one cannot distinguish between the SM Higgs field $H$ and $\Phi_{N / 2}$ unless they are distinguished by some additional symmetries. This category of models has been analyzed in Ref. [22], which we follow and impose a $Z_{2}$ symmetry on the new exotic fields $E, \Phi_{1 / 2}$, and $\Phi_{3 / 2}^{\prime}$. The corresponding Lagrangian and potential retain the general forms in Eqs. (2.1). Here we focus on the analysis of the DM candidate and explore the allowed region combining all the predictions and constraints as discussed before. Since the relic density from the kinetic term had been analyzed extensively in Ref. [23], we neglect the contributions from the potential terms with small couplings in order to satisfy the constraints of direct detection experiments such as LUX [24], XENON1T [25], and PandaX-II [26]. Therefore, we concentrate on the Yukawa coupling term $f$ with the mass range $m_{Z} / 2 \lesssim M_{X} \lesssim m_{W}{ }^{6}$. Moreover, we have to give a

\footnotetext{
${ }^{6}$ The lower bound comes from forbidding $Z \rightarrow 2 X$ decay which is strongly constrained by precision experiments, while the upper bound comes from the assumption that the kinetic terms does not contribute significantly to the relic density of DM.
} 
larger mass difference between $C P$-even and $C P$-odd bosons of $\phi^{0} \sim \phi_{R}+i \phi_{I}$, (either of which can be the $\mathrm{DM}$ ), in order to evade large cross sections via an interaction of $Z-\phi_{R}-\phi_{I}$, otherwise the coannihilation that gives a very small relic density. ${ }^{7}$ The relevant interaction of the DM $\phi_{R}=X$ is then given by

$$
-\mathcal{L}=\frac{f_{i a}}{\sqrt{2}} \bar{\ell}_{i} P_{R} E_{a} X+\text { H.c. }
$$

Then the dominant contribution is found to be the effective $\mathrm{d}$-wave coefficient after expanding the cross section in terms of the relative velocity. Its form is given by [28]

$$
d_{\mathrm{eff}}\left(2 X \rightarrow f_{i} \bar{f}_{j}\right) \approx \frac{M_{X}^{6}}{240 \pi}\left|\sum_{a=1}^{3} \frac{f_{i a} f_{a j}^{\dagger}}{\left(M_{X}^{2}+M_{E_{a}}^{2}\right)^{2}}\right|^{2},
$$

where $\left(f_{i}, f_{j}\right)=(e, \mu, \tau)$ run over all the charged-lepton mass eigenstates, and we have taken the charged-lepton masses in the final state to be zero. The resulting relic density is given by

$$
\Omega h^{2} \approx \frac{6.852 \times 10^{-9}}{\mathrm{GeV}^{2}} \frac{1}{d_{\mathrm{eff}}}
$$

where we have used several physical parameters given in Ref. [28]. The measured relic density is about 0.12 [29], and we apply the constraint as $0.11 \lesssim \Omega h^{2} \lesssim 0.13$ in our numerical analysis.

Since this mode is d-wave suppressed, so a rather large $f$ is required, which is in favor of generating a sizable $\Delta a_{\mu}$. Notice here that we have to replace $\phi$ by $\phi_{R} / \sqrt{2}$ and $\phi_{I} / \sqrt{2}$, due to the mass difference between $\phi_{R}$ and $\phi_{I}$. Hence all the general forms, except for the neutrino mass matrix, should be modified according to the replacement. For example, $F_{l f v}\left(M_{E_{a}}, m_{\phi}\right) \rightarrow\left[F_{l f v}\left(M_{E_{a}}, m_{\phi_{R}}\right)+F_{l f v}\left(M_{E_{a}}, m_{\phi_{I}}\right)\right] / 2$ in Eqs. (2.9), and (2.10), $F_{2,3}\left(E_{a}, \phi\right) \rightarrow\left[F_{2,3}\left(E_{a}, \phi_{R}\right)+\right.$ $\left.F_{2,3}\left(E_{a}, \phi_{I}\right)\right] / 2$ in Eq. (2.15), $G(\phi, \phi) \rightarrow\left[G\left(\phi_{R}, \phi_{R}\right)+\right.$ $\left.G\left(\phi_{I}, \phi_{I}\right)\right] / 2$ and $H(\phi) \rightarrow\left[H\left(\phi_{R}\right)+H\left(\phi_{I}\right)\right] / 2$ in Eq. (2.18).

\section{B. $N=3$}

The $N=3$ case is the minimal scenario without the need of additional symmetries. Several new terms are added as follows:

$$
-\mathcal{L}_{Y}^{\text {new }}=\kappa_{i j} \bar{e}_{i} P_{R} e_{j}^{c} k^{--}+\text {H.c., }
$$

\footnotetext{
${ }^{7}$ The typical mass difference is about $10 \%-20 \%$ of the DM mass [27], and the mass difference can arise from the additional term $\left(H^{\dagger} \Phi_{1 / 2}\right)^{2}+$ c.c..
}

$$
\begin{aligned}
V^{\text {new }}= & \mu_{k}^{2}\left|k^{++}\right|^{2}++\lambda_{k}\left|k^{++}\right|^{4}+\lambda_{H k}|H|^{2}\left|k^{++}\right|^{4} \\
& +\lambda_{\Phi k}\left|\Phi_{3 / 2}\right|^{2}\left|k^{++}\right|^{4}+\lambda_{\Phi^{\prime} k}\left|\Phi_{5 / 2}^{\prime}\right|^{2}\left|k^{++}\right|^{4} \\
& +\left[\mu\left(H^{T} \cdot \Phi_{\frac{3}{2}}\right) k^{--}+\text {c.c. }\right]+\left[\mu^{\prime}\left(H^{\dagger} \Phi_{\frac{5}{2}}\right) k^{--}+\text {c.c. }\right],
\end{aligned}
$$

where the terms $\kappa, \mu, \mu^{\prime}$ mainly contribute to the appropriate decays into the SM particles as mentioned above. The whole analyses have already been studied [6], and so we abbreviate this case for the moment.

$$
\text { C. } N=5
$$

In $N=5$ case, additional terms are given by

$$
\begin{aligned}
-\mathcal{L}_{Y}^{\text {new }}= & h_{a i} \bar{E}_{a} P_{R} e_{i} k^{++}+\kappa_{i j} \bar{e}_{i}^{c} P_{R} e_{j} k^{++}+\text {H.c., } \\
V^{\text {new }}= & \mu_{k}^{2}\left|k^{++}\right|^{2}+\mu\left[\left(H^{\dagger} \Phi_{5 / 2}\right) k^{++}+\text {c.c. }\right] \\
& +\lambda_{k}\left|k^{++}\right|^{4}+\lambda_{H k}|H|^{2}\left|k^{++}\right|^{4}+\lambda_{\Phi k}\left|\Phi_{5 / 2}\right|^{2}\left|k^{++}\right|^{4} \\
& +\lambda_{\Phi^{\prime} k}\left|\Phi_{7 / 2}^{\prime}\right|^{2}\left|k^{++}\right|^{4}+\text { H.c., }
\end{aligned}
$$

where the terms $h, \kappa, \mu$ mainly contribute to the appropriate decays into the SM particles. Especially, $h$ can also positively contribute to the muon anomalous magnetic moment as well as LFVs. The formula for $\mathcal{M}_{h}$ can be expressed in a similar way as the generic form of $\mathcal{M}_{f}$ in Eq. (2.9),

$\left(\mathcal{M}_{h}\right)_{i j} \approx-\sum_{a=1-3} \frac{h_{a j} h_{i a}^{\dagger}}{(4 \pi)^{2}}\left[2 F_{l f v}\left(M_{E_{a}}, m_{k}\right)+3 F_{l f v}\left(m_{k}, M_{E_{E a}}\right)\right]$,

where $F_{l f v}$ is defined in Eq. (2.11).

Flavor-changing leptonic $Z$ boson decays are modified due to the contribution of $h$, and the resulting form is given by

$$
\begin{aligned}
\operatorname{BR}\left(Z \rightarrow \ell_{i}^{-} \ell_{j}^{+}\right)= & \frac{G_{F}}{3 \sqrt{2} \pi} \frac{m_{Z}^{3} s_{w}^{4}(N+1)^{2}}{4\left(16 \pi^{2}\right)^{2} \Gamma_{Z}^{\text {tot }}} \\
& \times \mid \sum_{a=1}^{3} f_{i a} f_{j a}^{*}\left[F_{2}\left(E_{a}, \phi\right)+F_{3}\left(E_{a}, \phi\right)\right] \\
& +\sum_{b=1}^{3} g_{i b} g_{j b}^{*}\left[F_{2}\left(E_{b}, \phi^{\prime}\right)+F_{3}\left(E_{b}, \phi^{\prime}\right)\right] \\
& +\left.\sum_{c=1}^{3} h_{i c}^{\dagger} h_{j c}^{T}\left[F_{2}\left(E_{c}, k^{ \pm \pm}\right)+F_{3}\left(E_{c}, k^{ \pm \pm}\right)\right]\right|^{2} .
\end{aligned}
$$

Decay modes: Possible decay modes of the exotic particles are 


$$
\begin{array}{r}
\phi^{--} \underbrace{\rightarrow}_{f} E^{-3} \ell^{+}, \quad \phi^{\prime-4} \underbrace{\rightarrow}_{g} E^{-3} \ell^{-}, \\
E^{-3} \rightarrow 3 \ell^{-}:(E^{-3} \underbrace{\rightarrow}_{h} \ell^{-} k^{--}), \quad(k^{--} \underbrace{\rightarrow}_{\kappa} \ell^{-} \ell^{-}),
\end{array}
$$

where the upper component of the $\Phi_{5 / 2}$ doublet and the lower one of $\Phi_{7 / 2}^{\prime}$ mix with each other. Both always decay into $\phi^{--}$and/or $\phi^{\prime-4}$ via the kinetic terms, or they can directly decay into the SM leptons and $E^{ \pm 3}$ through $f$ and $g$ respectively, which are the same modes as the case $N=3$.

$$
\text { D. } N=7
$$

In $N=7$ case, additional terms are given by

$$
\begin{aligned}
-\mathcal{L}_{Y}^{\text {new }}= & \kappa_{i j} \bar{e}_{i}^{c} P_{R} e_{j} k^{++}+\text {H.c., } \\
V^{\text {new }}= & \mu_{k}^{2}\left|k^{++}\right|^{2}+\lambda_{1}\left[\left(H^{\dagger} \Phi_{9 / 2}^{\prime}\right) k^{--} k^{--}+\text {c.c. }\right] \\
& +\lambda_{2}\left[\left(H^{T} \cdot \Phi_{7 / 2}\right) k^{--} k^{--}+\text {c.c. }\right] \\
& +\lambda_{k}\left|k^{++}\right|^{4}+\lambda_{H k}|H|^{2}\left|k^{++}\right|^{4}+\lambda_{\Phi k}\left|\Phi_{7 / 2}\right|^{2}\left|k^{++}\right|^{4} \\
& +\lambda_{\Phi^{\prime} k}\left|\Phi_{9 / 2}^{\prime}\right|^{2}\left|k^{++}\right|^{4}+\text { H.c. },
\end{aligned}
$$

where the terms $\kappa, \lambda_{1}, \lambda_{2}$ mainly contribute to the appropriate decays into the SM particles.

Decay modes: Possible decay modes of the exotic particles are

$H_{1 / 2}^{-4}$ (Mixing state of $\left.\phi_{7 / 2}^{-4}-\phi_{9 / 2}^{\prime-4}\right) \underbrace{\rightarrow}_{\lambda_{1 / 2}, \kappa} 4 \ell^{-}\left(h_{\mathrm{SM}}\right)$,

where the other modes are the same as the general $N$.

$$
\text { E. } N=9
$$

In $N=9$ case, additional terms are given by

$$
\begin{aligned}
-\mathcal{L}_{Y}^{\text {new }}= & \kappa_{i j} \bar{e}_{i}^{c} P_{R} e_{j} k^{++}+\text {H.c., } \\
V^{\text {new }}= & \mu_{k}^{2}\left|k^{++}\right|^{2}+\lambda_{1}\left[\left(H^{\dagger} \Phi_{9 / 2}\right) k^{--} k^{--}+\text {c.c. }\right] \\
& +\lambda_{k}\left|k^{++}\right|^{4}+\lambda_{H k}|H|^{2}\left|k^{++}\right|^{4}+\lambda_{\Phi k}\left|\Phi_{9 / 2}\right|^{2}\left|k^{++}\right|^{4} \\
& +\lambda_{\Phi^{\prime} k}\left|\Phi_{11 / 2}^{\prime}\right|^{2}\left|k^{++}\right|^{4}+\text { H.c., }
\end{aligned}
$$

where the terms $\kappa, \lambda_{1}, \lambda_{2}$ mainly contribute to the appropriate decays into the SM particles.

Decay modes: Possible decay modes of the exotic particles are

$$
H_{1 / 2}^{-4}\left(\text { Mixing state of } \phi_{9 / 2}^{-4}-\phi_{11 / 2}^{\prime-4}\right) \underbrace{\rightarrow}_{\lambda_{1}, \kappa} 4 \ell^{-}\left(h_{\mathrm{SM}}\right),
$$

where the other modes are the same as the general $N$.

\section{NUMERICAL ANALYSIS OF $N=1-9$}

Here we discuss the features of the global analysis for $N=1$ and $N=3,5,7,9$ separately, because $N=1$ involves the constraint of the dark matter relic density.

$$
\text { A. } N=1
$$

The $N=1$ case is different from the other $N$ 's, because of the additional $Z_{2}$ symmetry imposed in order to distinguish between the Higgs field and the $\Phi_{1 / 2}$. Due to the $Z_{2}$ symmetry, the model has a dark matter candidate.

The dimensionless parameters are chosen to be scanned over the following moderate ranges without violating naturalness or perturbativity, as well as the mass parameters:

$$
\begin{aligned}
&\left|s_{2 \theta}\right| \leq 1, \quad(|\alpha|,|\beta|,|\gamma|) \in\left[10^{-15}, 10^{-5}\right],\left|f_{1 i}\right| \in\left[10^{-10}, 10^{-5}\right], \quad\left|f_{2 i}\right| \in[1,4 \pi], \\
&\left|f_{3 i}\right| \in\left[10^{-3}, 10^{-2}\right], \\
& M_{X} \in\left[m_{Z} / 2, m_{W}\right] \mathrm{GeV}, \\
& m_{\phi_{I}} \in\left[1.2 M_{X}, 150\right] \mathrm{GeV}, \\
& {\left[m_{H_{1}}, m_{H_{2}}, m_{\phi^{\prime}}\right] \in[100,150] \mathrm{GeV}, } \\
& M_{E_{1}} \in\left[1.2 M_{X}, 150\right] \mathrm{GeV}, \\
& M_{E_{2}} \in\left[M_{E_{1}}, 250\right] \mathrm{GeV}, \\
& M_{E_{3}} \in\left[M_{E_{2}}, 500\right] \mathrm{GeV},
\end{aligned}
$$

where we have assumed $m_{H_{1}} \leq m_{H_{2}} \leq m_{\phi^{\prime}}$, and $i=1 \sim 3$. We show a few plots of correlations among the observables or parameters in Fig. 4, where all the constraints as discussed above [neutrino oscillation data, LFVs, relic density with $0.11 \leq \Omega h^{2} \leq 0.13$, the oblique parameters, and $\left.\Delta a_{\mu} \leq(26.1+8) \times 10^{-10}\right]^{8}$ are satisfied.

One remarkable feature could be found in the first panel that a sizable $\Delta a_{\mu}\left(\Delta a_{\mu}>10 \times 10^{-10}\right)$ can be achieved in good agreement together with the current relic density of DM due to the d-wave suppression, where the mass of $M_{X}$ is between 60 and $80 \mathrm{GeV}$. In the next panel (with the red plot), one finds that the lighter region of $M_{X}$ and the heavier mass region of $M_{H_{1}}$ are excluded; $m_{H_{1}} \lesssim 113 \mathrm{GeV}$. This could mainly arise from the constraint of the oblique parameters; especially $T$, since the $T$ parameter requires the mass degeneracy between each component of $\Phi_{N / 2}$, and also the LFVs forbids the top-left region. In the third panel (purple plot), the left-bottom region is also ruled out by the bounds on LFVs. In the fourth (pink plot) and fifth (brown plot) panels, all modes of the flavor-changing

\footnotetext{
${ }^{8} \mathrm{We}$ are content with a positive $\Delta a_{\mu}$ even though it is not within the $2 \sigma$ range of the data.
} 

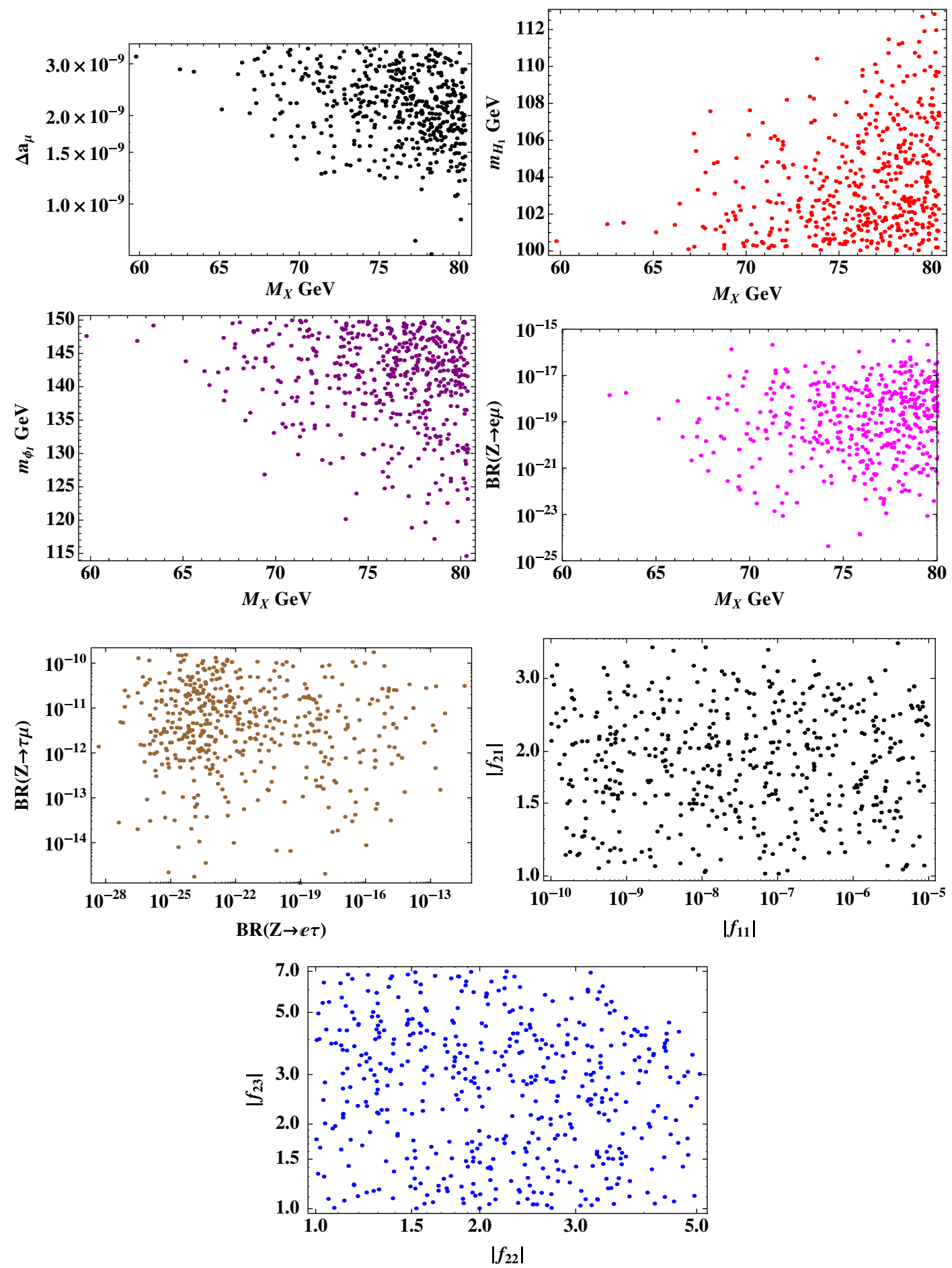

FIG. 4. $N=1$ case: Scatter plots between a pair of observables or parameters of the model, where $0.11 \leq \Omega h^{2} \leq 0.13$ is satisfied.

leptonic $Z$ boson decays are much below the current experimental bounds. This arises from the feature of the loop function which increases when the masses inside the loop are heavier. This result can be important in the other cases of $N$. In the sixth (black plot) and seventh (blue plot) panels, $\left|f_{2 i}\right|(i=1-3)$ are restricted to be $f_{21} \lesssim \sqrt{4 \pi}, f_{22} \lesssim 5$, and $f_{23} \lesssim 7$. These bounds originate from LFVs. Note here that all the other parameters run all over the range that we have taken in Eq. (4.3).

$$
\text { B. } N=3,5,7,9
$$

Next we investigate the case of $N=3,5,7,9$. The dimensionless parameters are scanned similarly to the $N=1$ case, and the mass parameters as well, 

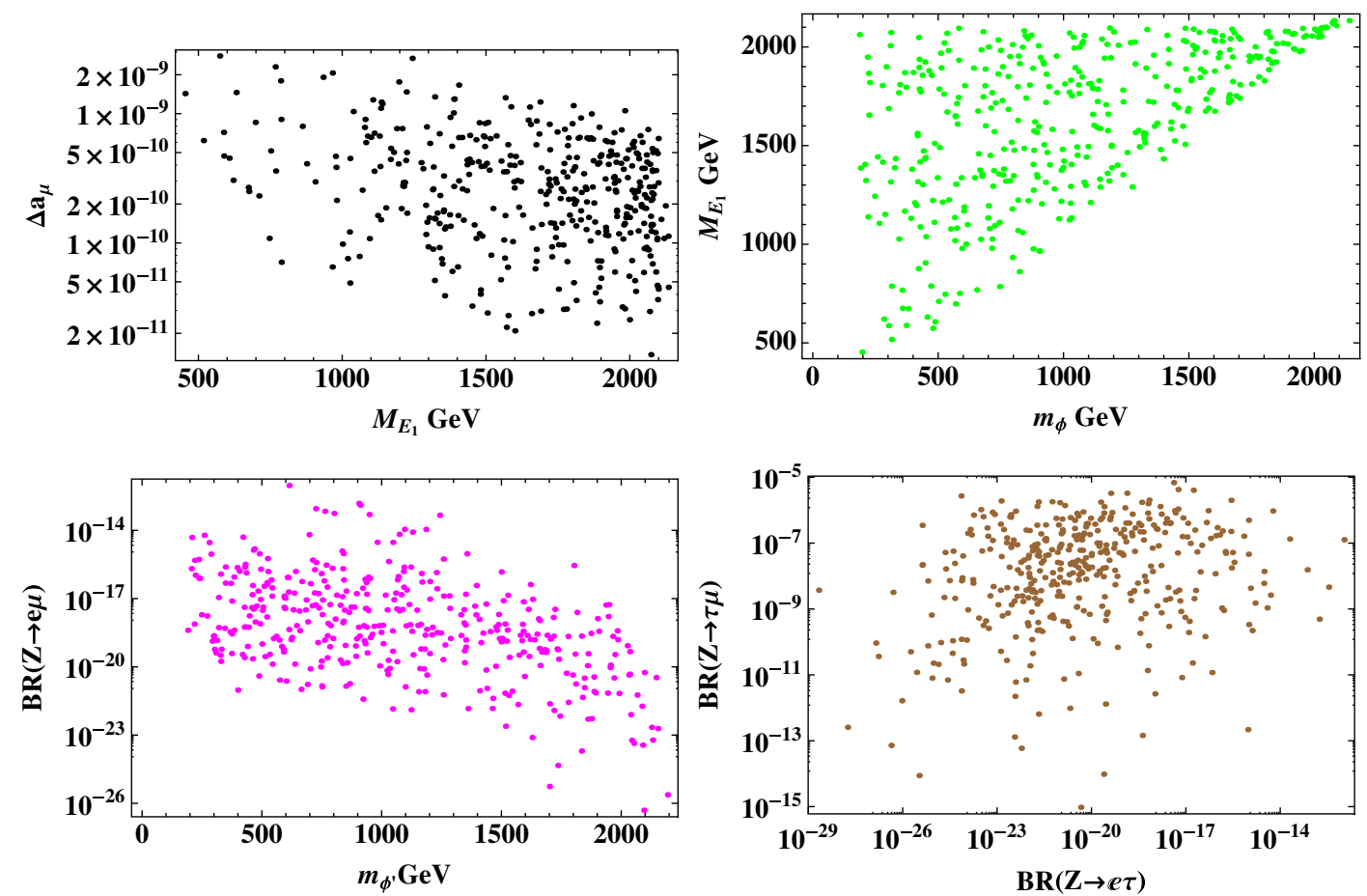

FIG. 5. $N=3$ case: Scatter plots between a pair of observables or parameters of the model.

$$
\begin{aligned}
& \left|f(h)_{1 i}\right| \in\left[10^{-10}, 10^{-5}\right], \quad\left|f(h)_{2 i}\right| \in[1,4 \pi], \\
& \left|f(h)_{3 i}\right| \in\left[10^{-3}, 1\right] \text {, }
\end{aligned}
$$
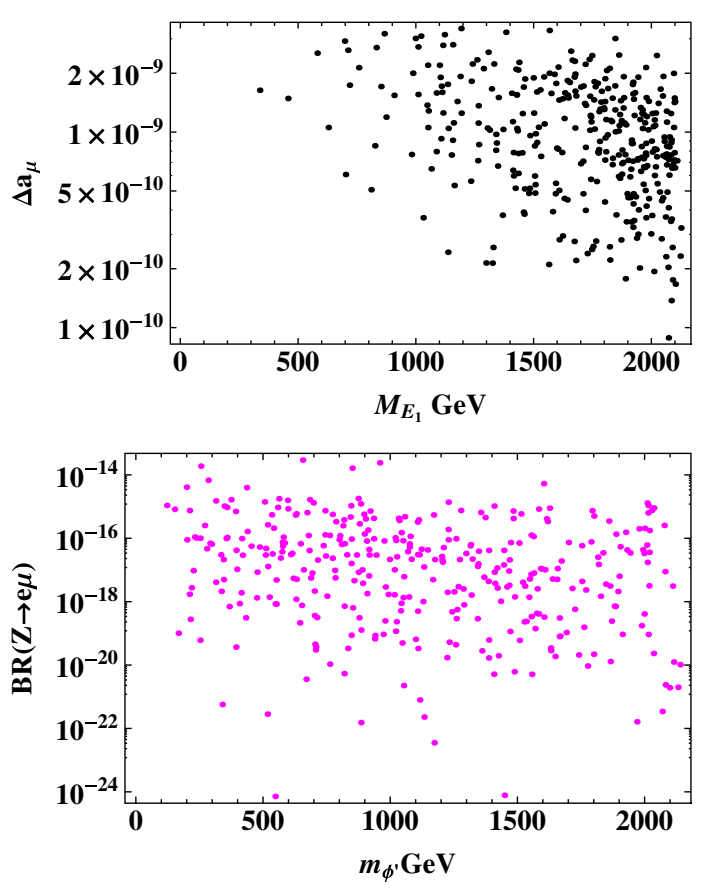

where we have assumed $m_{H_{1}} \leq m_{H_{2}} \leq m_{\phi} \leq m_{\phi^{\prime}}, M_{E_{1}} \leq$ $M_{E_{2}} \leq M_{E_{3}}, i=1-3$ and the others are the same as the case of $N=1$. Under these ranges, we show a number of plots in Figs. 5-8 for $N=3-9$, respectively. Several features are as follows:
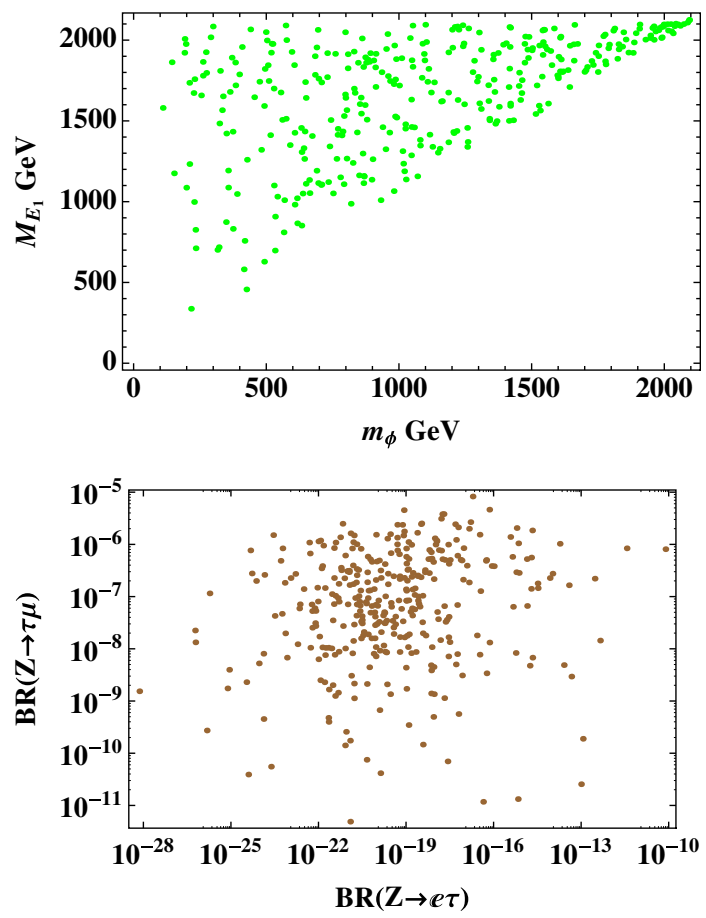

FIG. 6. $N=5$ case: Scatter plots between a pair of observables or parameters of the model. 

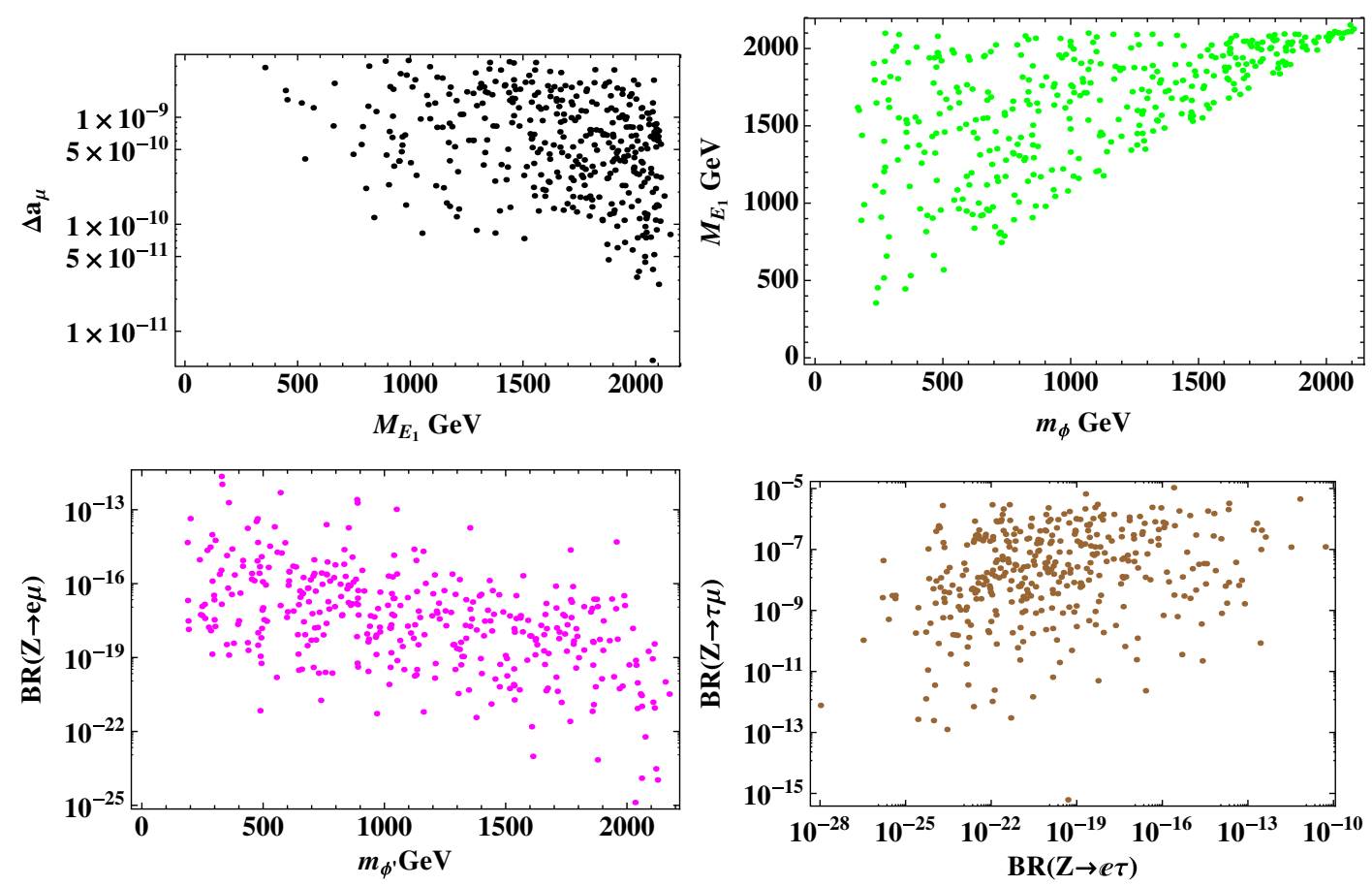

FIG. 7. $N=7$ case: Scatter plots between a pair of observables or parameters of the model.

(1) In the first panel of all Figs. 5-8, the majority of the allowed points can achieve positive $\Delta a_{\mu}$ larger than $1 \times 10^{-10}$ for $N=3-9$, and the allowed region that satisfies $\Delta a_{\mu}$ is wider when the number of $N$ increases.

(2) The upper-right panel of all Figs. 5-8 shows that $M_{E_{1}}>M_{\phi}$, whose mass hierarchy provides the larger value of loop function in the LFVs, because of the requirement of a positive $\Delta a_{\mu}$, as indicated in Eq. (2.9).

(3) In the bottom panels of all Figs. 5-8, the largest flavor-changing leptonic $Z$ decays are $Z \rightarrow \tau \mu$, which can be as large as $10^{-5}$, while the other two decay
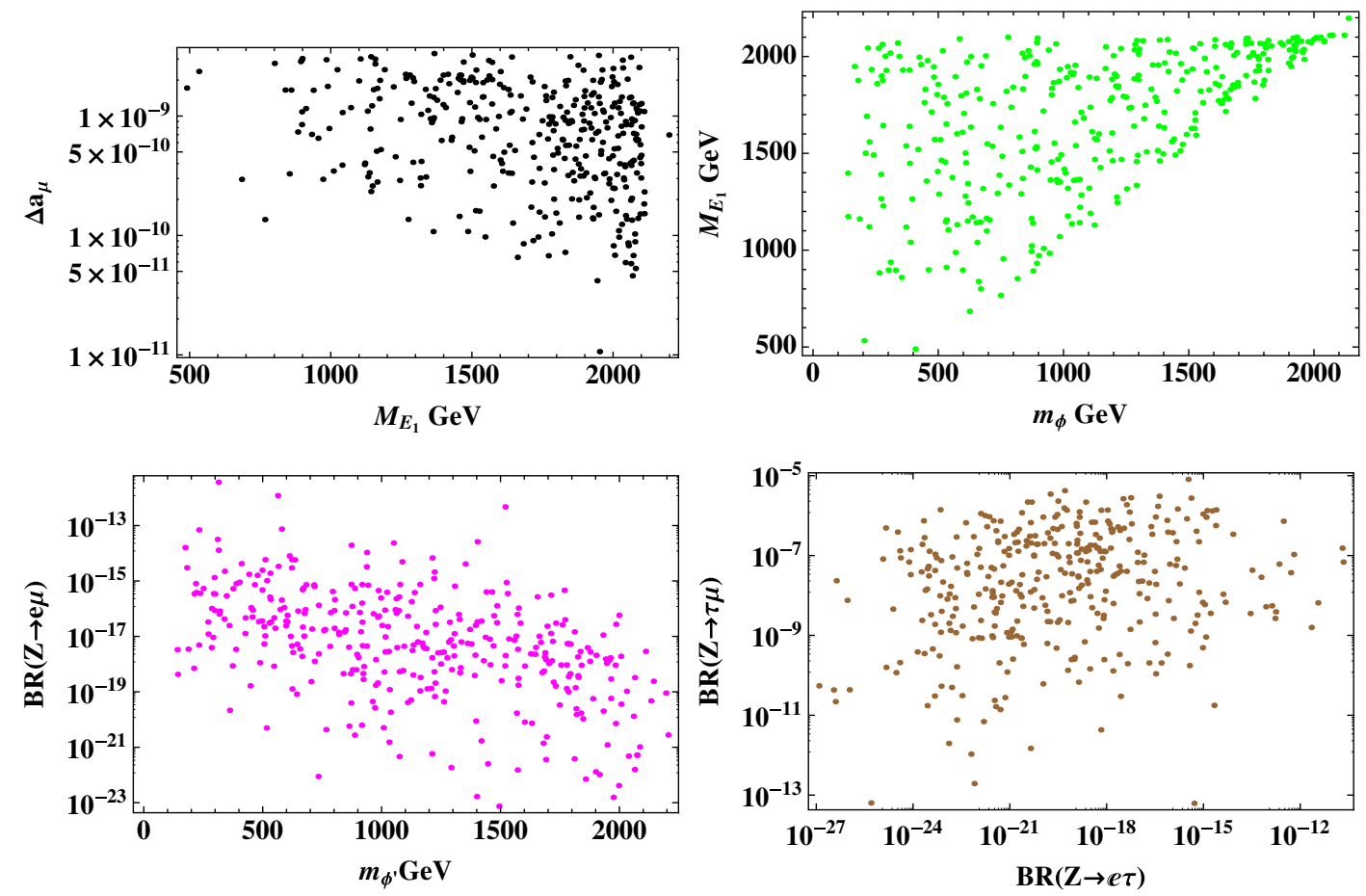

FIG. 8. $\quad N=9$ case: Scatter plots between a pair of observables or parameters of the model. 
branching ratios are many orders of magnitude below the current limits. The expected value for $\operatorname{BR}(Z \rightarrow$ $\tau \mu$ ) almost reaches the current experimental bound in Eq. (2.16) that could be tested by a Giga-Z type experiment at lepton colliders [15]. The large value mainly arises from the heavier masses inside the loop functions in addition to the larger Yukawa couplings. This is one of the important differences between $N=$ 1 and the other $N$ 's.

(4) We observe that $\operatorname{BR}\left(Z \rightarrow \tau^{ \pm} \mu^{\mp}\right)$ increases with an increase in $N$, which indeed links to larger $\Delta a_{\mu}$, with larger $\left|f_{2 i}\right|$. Similarly, larger $\operatorname{BR}\left(Z \rightarrow e^{ \pm} \mu^{\mp}\right)$ and $\operatorname{BR}\left(Z \rightarrow \tau^{ \pm} e^{\mp}\right)$ are obtained as $N$ increases. However, they are far below the current experimental bounds in Eq. (2.16).

(5) With increasing $N$, the number of allowed parameter-space points decreases. It mainly arises from the constraints of LFVs and the oblique parameters.

(6) For $N=5$ there are additional interactions among $k^{++}, E_{a}$ and $e_{i}$, which contribute to the lepton-flavor violation and $\Delta a_{\mu}$ in the same way as the $f$ terms in Eq. (2.9). Therefore, the behavior of the plots for $h_{i j}$ would be very similar to those of $f_{i j}$,

All the other parameters run all over the ranges that we have taken in Eq. (4.5), and the allowed mass region between $m_{H_{1}}-m_{H_{2}}$ is restricted by the $T$-parameter such that they are close to each other.

\section{COLLIDER SIGNALS}

We expect that the largest production rate of the exotic particles comes from pair production of the lightest fermions $E_{1}$. Drell-Yan (DY) production of $E_{1} \bar{E}_{1}$ occurs via $\gamma, Z$ exchanges. The interactions can be obtained from the kinetic term of the fermion $E_{1}$. Since $E_{1}$ is a singlet, the interactions with $\gamma$ and $Z$ are given by

$$
\mathcal{L}=-e \bar{E}_{1} \gamma^{\mu} Q_{E} E_{1} A_{\mu}+\frac{g s_{W}^{2}}{c_{W}} \bar{E}_{1} \gamma^{\mu} Q_{E} E_{1} Z_{\mu}
$$

where $s_{W}$ and $c_{W}$ are respectively the sine and cosine of the Weinberg angle, and $Q_{E}$ is the electric charge of the fermion $E_{1}$. The following applies for $N=1,3,5,7,9$ and $E=E_{1}$ for simplicity.

The square of the scattering amplitude, summed over spins, for $q\left(p_{1}\right) \bar{q}\left(p_{2}\right) \rightarrow E\left(k_{1}\right) \bar{E}\left(k_{2}\right)$ can be written as

$$
\begin{aligned}
\sum|\mathcal{M}|^{2}= & 4 e^{4} Q_{E}^{2}\left[\left(\hat{u}-M_{E}^{2}\right)^{2}+\left(\hat{t}-M_{E}^{2}\right)^{2}+2 \hat{s} M_{E}^{2}\right] \\
& \times\left\{\left|\frac{Q_{q}}{\hat{s}}-\frac{g_{L}^{q}}{c_{W}^{2}} \frac{1}{\hat{s}-m_{Z}^{2}}\right|^{2}+\left|\frac{Q_{q}}{\hat{s}}-\frac{g_{R}^{q}}{c_{W}^{2}} \frac{1}{\hat{s}-m_{Z}^{2}}\right|^{2}\right\},
\end{aligned}
$$

where $\hat{s}, \hat{t}, \hat{u}$ are the usual Mandelstam variables for the subprocess, and $g_{L, R}^{q}$ are the chiral couplings of quarks to the $Z$ boson. The subprocess differential cross section is given by

$$
\begin{aligned}
\frac{d \hat{\sigma}}{d \cos \hat{\theta}}= & \frac{\beta e^{4} Q_{E}^{2}}{96 \pi}\left[\left(\hat{u}-M_{E}^{2}\right)^{2}+\left(\hat{t}-M_{E}^{2}\right)^{2}+2 \hat{s} M_{E}^{2}\right] \\
& \times\left\{\left|\frac{Q_{q}}{\hat{s}}-\frac{g_{L}^{q}}{c_{W}^{2}} \frac{1}{\hat{s}-m_{Z}^{2}}\right|^{2}+\left|\frac{Q_{q}}{\hat{s}}-\frac{g_{R}^{q}}{c_{W}^{2}} \frac{1}{\hat{s}-m_{Z}^{2}}\right|^{2}\right\},
\end{aligned}
$$

where $\beta=\sqrt{1-4 M_{E}^{2} / \hat{s}}$, and $g_{L}^{q}=T_{3 q}-s_{W}^{2} Q_{q}, g_{R}^{q}=-s_{W}^{2} Q_{q}$, where $T_{3 q}$ is the third component of the isospin of $q$. This subprocess cross section is then folded with parton distribution functions to obtain the scattering cross section at the $p p$ collision level. The $K$ factor for the production cross sections is expected to be similar to the conventional DY process, which is approximately $K \simeq 1.3$ at the LHC energies. The production cross sections for $p p \rightarrow E_{1} \bar{E}_{1}$ at $\sqrt{s}=13 \mathrm{TeV}$ scale as $Q_{E}^{2}$, where $Q_{E}=(N+1) / 2$. In Ref. [6], the production cross sections for $E^{--} E^{++}$are shown, and so therefore for other $N=1,5,7,9$ can be easily estimated by simple scaling.

We can also derive the decay width of $E_{1}$ for a general $N$. The major decay modes are those via the terms $f_{i 1}$ and $g_{i 1}$ in the Lagrangian in Eq. (2.1). The decay modes are

$$
\begin{aligned}
E_{1}^{-(N+1) / 2} \rightarrow & \nu_{i} \phi_{N / 2}^{-(N+1) / 2}, & & \ell_{i}^{-} \phi_{N / 2}^{-(N-1) / 2}, \\
& \nu_{i} \phi_{N^{\prime} / 2}^{\prime-(N+1) / 2}, & & \ell_{i}^{+} \phi_{N^{\prime} / 2}^{-(N+3) / 2} .
\end{aligned}
$$

The decay width is given by

$$
\begin{aligned}
\Gamma\left(E_{1}\right)= & \frac{M_{E_{1}}}{32 \pi}\left\{\left(1-\frac{m_{\phi}^{2}}{M_{E_{1}}^{2}}\right) \sum_{i=1}^{3}\left|f_{i 1}\right|^{2}\right. \\
& \left.+\left(1-\frac{m_{\phi^{\prime}}^{2}}{M_{E_{1}}^{2}}\right) \sum_{i=1}^{3}\left|g_{i 1}\right|^{2}\right\} .
\end{aligned}
$$

Nevertheless, the contributions from $f_{i 1}$ dominate because of the requirement of the $\Delta a_{\mu}$.

Here we start with the detection of the final states for each case of $N=1,3,5,7,9$. We focus on the lightest state $E_{1}$.

$$
\text { A. } N=1
$$

The exotic scalar doublet fields take the form,

$$
\phi_{1 / 2}=\left(\begin{array}{c}
\phi_{1 / 2}^{+} \\
\phi_{1 / 2}^{0}
\end{array}\right), \quad \phi_{3 / 2}^{\prime}=\left(\begin{array}{c}
\phi_{3 / 2}^{++} \\
\phi_{3 / 2}^{\prime+}
\end{array}\right) .
$$

The neutral component $\phi_{1 / 2}^{0}$ is stable because of the $Z_{2}$ symmetry. The exotic fermion $E^{-}$can decay via the terms $f_{i 1}$ and $g_{i 1}$, 


$$
\begin{aligned}
-\mathcal{L} \supset & f_{i 1}\left(\bar{\nu}_{i} \phi_{1 / 2}^{+}+\bar{\ell}_{i} \phi_{1 / 2}^{0}\right) E_{1_{R}} \\
& +g_{i 1}\left(\bar{\nu}_{i} \phi_{3 / 2}^{\prime-}-\bar{\ell}_{i} \phi_{3 / 2}^{\prime--}\right) E_{1_{R}}^{c}+\text { H.c. }
\end{aligned}
$$

Assuming $|f| \gg|g|$ due to the requirement of $\Delta a_{\mu}$, the exotic fermion $E_{1}^{-}$decays via the term $f_{i 1}$ as

$$
E_{1}^{-} \rightarrow \nu \phi_{1 / 2}^{-}, \quad \ell^{-} \phi_{1 / 2}^{0},
$$

where $\phi_{1 / 2}^{-} \rightarrow \phi_{1 / 2}^{0} W^{*}$ and $\phi_{1 / 2}^{0}$ is the lightest exotic particle being stable. Therefore, Drell-Yan production of $E_{1}^{-} E_{1}^{+}$gives rise to a pair of charged leptons (not necessarily of the same flavor) plus missing energies.

\section{B. $N=3$}

This particular case has been given in detail in Ref. [6]. In summary, the exotic fermion pair $E_{1}^{--} E_{1}^{++}$is produced via the Drell-Yan process. The fermion $E_{1}^{--}$decays via the terms $f_{i 1}$ and $g_{i 1}$,

$$
\begin{aligned}
-\mathcal{L} \supset & f_{i 1}\left(\bar{\nu}_{i} \phi_{3 / 2}^{++}+\bar{\ell}_{i} \phi_{3 / 2}^{+}\right) E_{1_{R}}^{--} \\
& +g_{i 1}\left(\bar{\nu}_{i} \phi_{5 / 2}^{\prime--}-\bar{\ell}_{i} \phi_{5 / 2}^{\prime 3-}\right) E_{1_{R}}^{c}+\text { H.c. }
\end{aligned}
$$

The decays via the dominant $f_{i 1}$ term are

$$
E_{1}^{--} \rightarrow \ell^{-} \phi_{3 / 2}^{-}, \quad \nu \phi_{3 / 2}^{--},
$$

followed by (via the mixing with scalar $k^{--}$)

$$
\begin{aligned}
\phi_{3 / 2}^{--} & \rightarrow \ell^{-} \ell^{-} \\
\phi_{3 / 2}^{-} \rightarrow \phi_{3 / 2}^{--} W^{+} & \rightarrow \ell^{-} \ell^{-} W^{+} .
\end{aligned}
$$

Each $E_{1}^{--}$decays into two or four charged leptons. Therefore, the final state can consist of 4,6 , or 8 charged leptons plus missing energies.

\section{C. $N=5$}

This case is similar to $N=3$. The relevant terms in the Lagrangian responsible for the decay of the exotic fermion $E^{3-}$ are

$$
\begin{aligned}
-\mathcal{L} \supset & f_{i 1}\left(\bar{\nu}_{i} \phi_{5 / 2}^{3+}+\bar{\ell}_{i} \phi_{5 / 2}^{2+}\right) E_{1_{R}}^{3-}+g_{i 1}\left(\bar{\nu}_{i} \phi_{7 / 2}^{\prime 3-}-\bar{\ell}_{i} \phi_{7 / 2}^{\prime 4-}\right) E_{1_{R}}^{c} \\
& +h_{1 i} \bar{E}_{1} P_{R} \ell_{i} k^{--}+\kappa_{i j} \bar{e}_{i}^{c} P_{R} e_{j} k^{++}+\text {H.c., }
\end{aligned}
$$

in which $\phi_{5 / 2}^{2+}$ and $k^{++}$mix. The exotic fermion $E_{1}^{3-}$ so produced will decay via

$$
\begin{aligned}
& E_{1}^{3-} \rightarrow \nu \phi_{5 / 2}^{3-} \rightarrow \nu \phi_{5 / 2}^{2-} W^{-} \rightarrow \nu \ell^{-} \ell^{-} W^{-} \\
& E_{1}^{3-} \rightarrow \ell^{-} \phi^{2-}, \quad \ell^{-} k^{--} \rightarrow \ell^{-} \ell^{-} \ell^{-} .
\end{aligned}
$$

Each $E_{1}^{3-}$ decays into 3 charged leptons, without or with small missing energy. Therefore, the final state of $E^{3-} E^{3+}$ pair production consists of 6 charged leptons, mainly without missing energies.

\section{D. $N=7$}

In this case, there are additional terms in the Lagrangian that allow the quartic-charged $\phi$ or $\phi^{\prime}$ to decay into a pair of $k^{++} k^{++}$, which further decay into 4 charged leptons.

$$
\begin{aligned}
-\mathcal{L} \supset & f_{i 1}\left(\bar{\nu}_{i} \phi_{7 / 2}^{4+}+\bar{\ell}_{i} \phi_{7 / 2}^{3+}\right) E_{1_{R}}^{4-}+g_{i 1}\left(\bar{\nu}_{i} \phi_{9 / 2}^{4-}-\bar{\ell}_{i} \phi_{9 / 2}^{\prime 5-}\right) E_{1_{R}}^{c} \\
& +\lambda_{1} v \phi^{\prime++} k^{--} k^{--}+\lambda_{2} v \phi^{4+} k^{--} k^{--} \\
& +\kappa_{i j} \bar{e}_{i}^{c} P_{R} e_{j} k^{++}+\text {H.c. }
\end{aligned}
$$

Each exotic fermion $E_{1}^{4-}$ decays via

$$
\begin{aligned}
E_{1}^{4-} & \rightarrow \nu \phi_{7 / 2}^{4-} \rightarrow \nu k^{--} k^{--} \rightarrow \nu \ell^{-} \ell^{-} \ell^{-} \ell^{-} \\
E_{1}^{4-} & \rightarrow \ell^{-} \phi_{7 / 2}^{3-} \rightarrow \ell^{-} \phi_{7 / 2}^{4-} W^{+} \rightarrow \ell^{-} k^{--} k^{--} W^{+} \\
& \rightarrow \ell^{-} \ell^{-} \ell^{-} \ell^{-} \ell^{-} W^{+} .
\end{aligned}
$$

Thus, each $E_{1}^{4-}$ can decay into 4 or 6 charged leptons. Therefore, the final state of $E_{1}^{4-} E_{1}^{4+}$ production consists of 8,10 , or 12 charged leptons.

\section{E. $N=9$}

In this case, there is one additional term in the Lagrangian that allows the quartic-charged $\phi$ to decay into a pair of $k^{++} k^{++}$, which further decay into 4 charged leptons.

$$
\begin{aligned}
-\mathcal{L} \supset & f_{i 1}\left(\bar{\nu}_{i} \phi_{9 / 2}^{5+}+\bar{\ell}_{i} \phi_{9 / 2}^{4+}\right) E_{1_{R}}^{5-} \\
& +g_{i 1}\left(\bar{\nu}_{i} \phi_{11 / 2}^{5-}-\bar{\ell}_{i} \phi_{11 / 2}^{6-}\right) E_{1_{R}}^{c} \\
& +\lambda_{1} v \phi_{9 / 2}^{4+} k^{--} k^{--}+\kappa_{i j} \bar{e}_{i}^{c} P_{R} e_{j} k^{++}+\text {H.c. }
\end{aligned}
$$

Each exotic fermion $E_{1}^{5-}$ decays via

$E_{1}^{5-} \rightarrow \nu \phi_{9 / 2}^{5-} \rightarrow \nu \phi_{9 / 2}^{4-} W^{-} \rightarrow \nu k^{--} k^{--} W^{-} \rightarrow \nu \ell^{-} \ell^{-} \ell^{-} \ell^{-} W^{-}$ $E_{1}^{5-} \rightarrow \ell^{-} \phi_{9 / 2}^{4-} \rightarrow \ell^{-} \ell^{-} \ell^{-} \ell^{-} \ell^{-}$.

Thus, each $E^{5-}$ decays into 5 charged leptons. Therefore, the final state of $E_{1}^{5-} E_{1}^{5+}$ production consists of 10 charged leptons.

\section{CONCLUSIONS}

We have generalized a radiative neutrino-mass model by introducing 3 generations of fermion pairs $E^{-(N+1) / 2} E^{+(N+1) / 2}$ and a couple of multicharged bosonic doublet fields $\Phi_{N / 2}, \Phi_{N / 2+1}$, where $N=1,3,5,7,9$. We have shown that the model can satisfy the neutrino masses and oscillations, lepton-flavor violations, the oblique parameters, and the $\Delta a_{\mu}$. We also made predictions for 
the collider signatures of the model. In general, searches for multicharged leptons in the final state, not necessarily of the same flavor, are interesting probes of the model.

We offer a few more comments as follows:

(1) For $N=1$, where there is an additional $Z_{2}$ symmetry to distinguish the Higgs field from $\Phi_{1 / 2}$, there is a possible dark matter candidate. On the other hand, for $N=3,5,7,9$ cases we require extra terms in the Lagrangian in order to make sure no stable charged particles remain. The real part of the neutral component of $\phi_{1}$ serves as the dark matter, and its mass is around $60-80 \mathrm{GeV}$, in order to satisfy the relic density of the Universe.

(2) In order to give large positive contributions to the $\Delta a_{\mu}$ the relevant components in $f$, such as $f_{21}, f_{22}$ and $f_{23}$, have to be large enough. On the other hand, other Yukawa couplings are in general very small because of lepton-flavor violation constraints.
(3) The largest flavor-changing leptonic $Z$ decays is $Z \rightarrow \tau \mu$, which can have a branching ratio as large as $10^{-5}$. It can be tested in the Giga-Z option in the future $e^{+} e^{-}$colliders.

(4) The mass splitting among the components in $\Phi$ doublet fields is restricted because of the $T$ parameters.

(5) Drell-Yan production of exotic fermion pairs would give rise to multicharged leptons in the final state. In general, a larger $N$ would give more charged leptons in the final state.

\section{ACKNOWLEDGMENTS}

We thank Chang-Hun Lee for discussion. This work was supported by the Ministry of Science and Technology of Taiwan under Grant No. MOST-105-2112-M-007028-MY3.
[1] T. Yanagida, Prog. Theor. Phys. 64, 1103 (1980); R. N. Mohapatra and G. Senjanovic, Phys. Rev. Lett. 44, 912 (1980); J. Schechter and J. W. Valle, Phys. Rev. D 25, 774 (1982).

[2] R. N. Mohapatra, Phys. Rev. Lett. 56, 561 (1986); R. N. Mohapatra and J. W. F. Valle, Phys. Rev. D 34, 1642 (1986).

[3] A. Zee, Phys. Lett. 93B, 389 (1980); 95B, 461(E) (1980).

[4] K. S. Babu, Phys. Lett. B 203, 132 (1988).

[5] E. Ma, Phys. Rev. D 73, 077301 (2006).

[6] K. Cheung and H. Okada, Phys. Lett. B 774, 446 (2017).

[7] H. Okada and Y. Orikasa, Phys. Rev. D 94, 055002 (2016).

[8] D. V. Forero, M. Tortola, and J. W. F. Valle, Phys. Rev. D 90 , 093006 (2014).

[9] K. A. Olive et al. (Particle Data Group), Chin. Phys. C 38, 090001 (2014).

[10] A. M. Baldini et al. (MEG Collaboration), Eur. Phys. J. C 76, 434 (2016).

[11] B. Aubert et al. (BABAR Collaboration), Phys. Rev. Lett. 104, 021802 (2010).

[12] K. Hagiwara, R. Liao, A. D. Martin, D. Nomura, and T. Teubner, J. Phys. G 38, 085003 (2011).

[13] C. W. Chiang, H. Okada, and E. Senaha, Phys. Rev. D 96, 015002 (2017).

[14] C. Patrignani et al. (Particle Data Group), Chin. Phys. C 40, 100001 (2016).

[15] J. A. Aguilar-Saavedra et al. (ECFA/DESY LC Physics Working Group), arXiv:hep-ph/0106315.
[16] K. Cheung, T. Nomura, and H. Okada, Phys. Rev. D 94, 115024 (2016).

[17] K. Cheung, H. Okada, and Y. Orikasa, arXiv:1706.02084.

[18] M. Baak, M. Goebel, J. Haller, A. Hoecker, D. Kennedy, R. Kogler, K. Mönig, M. Schott, and J. Stelzer, Eur. Phys. J. C 72, 2205 (2012).

[19] S. Dawson and W. Yan, Phys. Rev. D 79, 095002 (2009).

[20] P. Ko, T. Nomura, H. Okada, and Y. Orikasa, Phys. Rev. D 94, 013009 (2016).

[21] S. Kanemura, K. Nishiwaki, H. Okada, Y. Orikasa, S. C. Park, and R. Watanabe, Prog. Theor. Exp. Phys. 2016, 123B04 (2016).

[22] M. Aoki, S. Kanemura, and K. Yagyu, Phys. Lett. B 702, 355 (2011); 706, 495 (2012).

[23] T. Hambye, F.-S. Ling, L. Lopez Honorez, and J. Rocher, J. High Energy Phys. 07 (2009) 090; 05 (2010) 66.

[24] D. S. Akerib et al. (LUX Collaboration), Phys. Rev. Lett. 118, 021303 (2017).

[25] E. Aprile et al. (XENON Collaboration), Phys. Rev. Lett. 119, 181301 (2017).

[26] X. Cui et al. (PandaX-II Collaboration), Phys. Rev. Lett. 119, 181302 (2017).

[27] K. Griest and D. Seckel, Phys. Rev. D 43, 3191 (1991).

[28] T. Nomura and H. Okada, arXiv:1707.06083 [Phys. Rev. D (to be published)].

[29] C. Patrignani et al. (Particle Data Group), Chin. Phys. C 40, 100001 (2016) and 2017 update. 